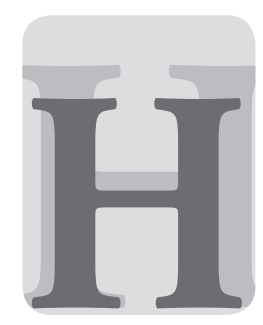

\title{
ACIA LA IDENTIFICACIÓN DE DIMENSIONES RELEVANTES RELACIONADAS CON EL APRENDIZAJE ORGANIZACIONAL
}

\section{Towards the identification of relevant dimensions related to organizational learning}

Adriana Norma Fassio* María Gabriela Rutty **

* Doctora en la Universidad de Buenos Aires, orientación Antropología Social. Magíster en Gerontología Social. Docente e investigadora, IADCOM- Instituto de Investigaciones en Administración, Contabilidad y Métodos Cuantitativos para la Gestión Facultad de Ciencias Económicas, Universidad de Buenos Aires, Caba - Argentina. E-mail: adriana.fassio@economicas.uba.ar

** Doctora en la Universidad de Buenos Aires, orientación Administración. Magíster en Administración Pública. Docente e investigadora. Facultad de Ciencias Económicas, Universidad de Buenos Aires, Caba - Argentina. E-mail: irutty@econ.uba.ar

Fecha de recepción: 16 de marzo de 2016

Fecha de aceptación: 18 de septiembre de 2016

Cómo citar / How to cite

Fasio, A. N. y Rutty, M. G. (2017). Hacia la identificación de dimensiones relevantes relacionadas con el aprendizaje organizacional. trilogía Ciencia Tecnología Sociedad, 9(16), 85- 99. 
Resumen: en este trabajo se realiza una revisión de los instrumentos utilizados para indagar sobre el aprendizaje organizacional, a fin de proponer una guía de relevamiento. En el campo del diagnóstico del aprendizaje organizacional prima la investigación a partir de escalas que dan cuenta de la opinión de los informantes sobre las dimensiones en las que se operacionaliza el aprendizaje organizacional, en tanto que son mucho menos frecuentes los abordajes cualitativos que intentan, a partir de un proceso inductivo, abordar el tema con la mirada de los actores. Partiendo de la identificación de las dimensiones consideradas y su operacionalización, así como de las técnicas puestas en juego para llevar adelante el relevamiento de la información, se realiza una propuesta de preguntas de investigación orientadas a identificar los procesos, obstáculos, facilitadores e impactos originados en los procesos de aprendizaje organizacional, como espacios en los que se recupera e incorpora en las rutinas organizacionales la experiencia adquirida en la resolución de los problemas que se enfrentan.

Palabras clave: dimensiones del aprendizaje organizacional, facilitadores y obstáculos del aprendizaje organizacional, instrumentos de relevamiento del aprendizaje organizacional.

Abstract: the aim of the paper is to review the tools used to investigate organizational learning in order to propose a survey guide. When diagnosing an organizational learning, investigation plays a key role. By incorporating various levels, an investigation takes into account the views of participants on the dimensions of the organizational learning. On the other hand studies that use a qualitative approach to this topic are less common when it comes to include an inductive process from the perspective of the actors involved in learning process. Based on the identification and operationalization of the dimensions considered, as well as the techniques carried out in the survey of information, this paper presents several research questions designed to identify the processes, barriers, facilitators, and impacts originated within the organizational learning processes, as opportunities to recover and include among the organizational routines lessons learned in solving the problems the organizations need to face.
Keywords: organizational learning, facilitators and obstacles of organizational learning, organizational learning research tools.

\section{INTRODUCCIÓN}

El fenómeno del Aprendizaje Organizacional (AO) ha sido considerado por muchos de los autores del campo del análisis organizacional y de la administración como un aspecto estratégico en la dirección de organizaciones, sin embargo, persisten múltiples interpretaciones acerca de los elementos componentes del fenómeno.

El propósito de este artículo es realizar un relevamiento de diversas formas de abordaje del fenómeno, que aportan a la formulación de un protocolo de preguntas sobre el aprendizaje organizacional. Con este objeto, se realiza un análisis descriptivo de las dimensiones conceptuales e indicadores que utilizan los autores seleccionados que abordan el estudio en forma empírica.

En un recorrido sobre la literatura existente, Ramírez y Zapata (2011) describen los principales exponentes sobre aprendizaje organizacional y las barreras que lo impiden. Para ello ordenan la producción teórica en dos grandes grupos a) trabajos descriptivos y b) trabajos prescriptivos.

La producción de tipo descriptiva manifiesta una preocupación más acentuada en la caracterización del aprendizaje organizacional. Son trabajos de tipo académico, donde se indaga acerca de las distintas dimensiones del fenómeno y algunas de estas producciones incluyen trabajo empírico. Argyris (1999) aporta los conceptos de aprendizaje de bucle simple y doble, a partir de considerar el aprendizaje en función de la detección y corrección de errores en las organizaciones. Los aprendizajes están determinados tanto a nivel individual como organizacional por las teorías expuestas o adaptadas desde la coincidencia o no entre lo que las personas dicen y lo que la personas hacen (Gore y Mazzini, 2010). En esta dirección se identifican dos tipos de aprendizajes: adaptativo y generativo (Ramírez y Zapata, 2011).

El aprendizaje adaptativo es un proceso de configuración estructural adaptable a las decisiones de sus directivos y, en consecuencia, a los cambios del 
entorno de la organización. El aprendizaje generativo es el que realizan las organizaciones a partir de una lectura adecuada del entorno, de manera que puedan adelantarse a sus competidores a través de diversas formas de adquirir, compartir, almacenar y utilizar el conocimiento por medio de las nuevas tecnologías (Rocha, 2013) y de los procesos desarrollados al interior de la organización (este tipo de aprendizaje corresponde a una ventaja competitiva). La dimensión descriptiva pone especial énfasis en los elementos que inhiben el proceso de aprendizaje llamados rutinas defensivas (Argyris, 1999; Senge, 2005).

El aprendizaje organizacional se encuentra determinado por la cultura organizacional, en particular los correspondientes al nivel de las presunciones básicas y los valores (Schein, 1992).

Los trabajos prescriptivos ponen el foco en cómo gestionar el aprendizaje en las organizaciones, considerándolo como una ventaja comparativa. De esta manera, su preocupación se orienta a la enunciación de una serie de ejes para promover el aprendizaje organizacional. Mucho de este tipo de literatura, mayormente de gestión, parte de la consideración de la existencia del fenómeno como un hecho y la preocupación no está centrada en la descripción del fenómeno, sino en la enunciación de las condiciones necesarias para generar el aprendizaje organizacional. Algunos de estos trabajos presentan también estudios empíricos.

Desde esta perspectiva se presta especial atención a las relaciones entre el proceso de aprendizaje organizacional y la relación entre individuosorganización-entorno. El escenario de aprendizaje está determinado por los continuos cambios del entorno, y es por ello que las organizaciones tienden a formular estrategias y configurar estructuras para hacer frente a dichos cambios. Ramírez y Zapata (2011) exponen la propuesta de Senge (2005), quien define el proceso de aprendizaje organizacional a partir de la adquisición de cinco disciplinas: dominio personal, modelos mentales, visión compartida, aprendizaje en equipo y pensamiento de sistema. En esta perspectiva se sostiene que existe una fuerte relación entre el aprendizaje individual y el organizacional y que las organizaciones aprenden a través de sus miembros (Antonacopoulou, 2006a, 2006b).

Algunos autores avanzan en identificar niveles de aprendizaje en las organizaciones (Garzón Castillón y Fischer, 2010; Rostro Hernández y Solís Hernández, 2015). Entre ellos, Garzón Castillón y Fisher reconocen cuatro niveles: 1) el que se gesta en los individuos, como una de las manifestaciones de su compromiso con la organización, como agentes de cambio en ella; 2) el que se da en el nivel de los grupos (dado por las estrategias de cooperación entre las personas para conseguir objetivos comunes); 3) el que se da a nivel de la organización, en la medida en que la organización en su totalidad aprende al recibir retroalimentación del medio, adelantándose a los cambios ; y finalmente, 4) a nivel interorganizacional, dado que las personas aprenden como parte de sus actividades diarias, cuando interaccionan con otros y con el mundo exterior, y este aprendizaje conlleva a la generación de innovación sobre la base de la confianza, percepción del riesgo, poder y vínculos. Por otro lado, se hace hincapié en la relevancia del aprendizaje no solo a nivel organizacional sino también en el marco de los microprocesos de aprendizaje, como parte de la transición de lo individual a lo colectivo (Rúas y Antonello, 2003).

Lo más habitual en los artículos relacionados con el estado del arte de la temática del aprendizaje organizacional son los debates vinculados con la conceptualización teórica; por el contrario, no son muchos los artículos en los que se hace referencia a las dimensiones, variables, indicadores y su definición conceptual a partir de los cuales se investigó y midió el aprendizaje.

Asimismo, son escasos los trabajos en donde se investiga la capacidad y las estrategias de aprendizaje en el sector público estatal/ no estatal (Pereira y Grau, 1998), ya que en líneas generales la bibliografía toma como unidad de análisis las organizaciones del sector privado con fines de lucro y relaciona el aprendizaje con la eficiencia y la innovación, cuyo indicador predilecto es la rentabilidad. En nuestro trabajo consideramos la creación de valor, como la capacidad de mejorar la calidad de vida de la población objetivo de su quehacer, como uno de 
los objetivos de la incorporación de conocimiento a partir de la resolución de los problemas que enfrentan las organizaciones públicas (Moore, 2006).

88 A continuación se presenta una revisión de algunos trabajos orientados a investigar el $\mathrm{AO}$, tratando de retomar algunos de los énfasis que presentan los autores revisados, tales como: aquellos que indagan sobre los factores que permiten o promueven el aprendizaje organizacional, los que estudian el proceso de aprendizaje organizacional, los que analizan el aprendizaje junto con los cambios del entorno organizacional, los que analizan el fenómeno del rendimiento del aprendizaje organizacional, las relaciones entre el aprendizaje individual y organizacional, el fracaso en el aprendizaje y aquellos que han explorado los niveles de aprendizaje organizacional.

\section{FACTORES QUE PROMUEVEN 0 PERMITEN EL APRENDIZAJE ORGANIZACIONAL}

Zárate (2007) realiza una revisión de los principales instrumentos de diagnóstico de aprendizaje organizacional y rescata el cuestionario realizado por Watkins y Marsick (1998) (Marsick y Watkins, 2003) denominado Dimension of the Learning Organization Questionnaire (DLOQ), con el objetivo de medir la cultura de aprendizaje de una organización que aprende. Identifica siete dimensiones: a) creación de oportunidades de aprendizaje continuo, es decir, que el trabajo esté diseñado de modo tal que las personas puedan aprender en el trabajo y que existan oportunidades de aprendizaje continuo y crecimiento; b) promoción de la indagación y el diálogo como espacios en que las personas puedan expresar sus puntos de vista e indagar en los pensamientos de los demás, cuestionar y experimentar; c) animación a la colaboración y el trabajo en equipo como instancias de acceso a diversos modos de pensamiento; d) creación de sistemas para capturar y compartir el aprendizaje en la medida en que existen sistemas de alta y baja tecnología para compartir los aprendizajes con acceso y con sostenimiento a través del tiempo; e) empoderamiento de las personas hacia una visión colectiva en la medida en que los integrantes compartan y se apropien de una visión conjunta que se acerque a los niveles de decisión; f) conexión de la organización con su medio ambiente, de modo tal que los miembros puedan identificar los efectos de su trabajo en la organización; y, g) liderazgo estratégico para el aprendizaje con el fin de obtener mejores resultados.

Ali, Pascoe y Warne (2002) también se centran en los factores que permiten el aprendizaje, y presentan propuesta de investigación exploratoria y los hallazgos de la investigación realizada por la Enterprise Social Learnig Architecture (ESLA) sobre los procesos de aprendizaje, en dos áreas del Departamento de Defensa de Australia. La investigación intentó identificar los factores que permiten y facilitan el aprendizaje social. Afirman que el aprendizaje social ocurre hacia el interior de un grupo/organización en un contexto (conocimiento situado) y supone: a) la capacidad de la organización para generar nuevo conocimiento que le permita ajustarse a situaciones inesperadas y reaccionar creativamente a ellas; y b) los procesos a partir de los cuales conocimiento y práctica son transmitidos a través del tiempo y en diversas situaciones de trabajo. En este estudio piloto se utilizaron técnicas etnográficas, tales como observación y cuestionarios en el campo: se realizaron 59 entrevistas y una encuesta para triangular la información, métodos e investigadores. El equipo ESLA identificó siete categorías básicas que constituyen los procesos y estrategias que facilitan el aprendizaje social. Por lo tanto, el aprendizaje organizacional se facilita cuando existe: 1) entidad común: alineación con las metas, identidad cultural, identidad de género; lenguaje, moral y diseño del espacio de trabajo (físico y espacial); 2) solución de los problemas: como una oportunidad de aprendizaje. Habilitadores: redes, improvisación, percepción de la organización, comprensión sistémica y tiempo para preguntar y reflexionar; 3) construcción de equipos: trabajar en conjunto y comprender lo que cada miembro está tratando de hacer. Habilitadores: liderazgo, espíritu de equipo, gestión del rendimiento, reconocimiento público y sistema de recompensas; uso del humor y diseño de los espacios de trabajo; 4) acceso a la información: acceso fácil a la información corporativa en cualquier formato. Aspectos asociados: mantenimiento de los registros, creación de redes, reuniones, y la infraestructura en tecnologías de la información; 5) desarrollo de las capacidades personales: trayectorias 
de carrera, desarrollo profesional, entrenamiento, direccionamiento, promoción y tutorías en la carrera profesional; 6) comunicación: esencial para favorecer el aprendizaje:-clima comunicacional, circulación de información formal e informal, tiempo para inquirir y reflexionar, uso del humor, del lenguaje y el diseño del espacio de trabajo; y 7) inducción y enculturación (adquisición gradual de las características de la cultura organizacional): facilita el aprendizaje social en la medida que provee una base a partir de la cual el individuo es totalmente productivo. Factores asociados: oportunidad e integralidad de los procesos; sistemas de tutoría, traspasos de la información, paquetes de información y entrenamiento.

Los autores Ali, Pascoe y Warne (2002) sugieren que el desarrollo de los sistemas de aprendizaje requiere una comprensión de los aspectos culturales e interpersonales que prevalecen en los ambientes de trabajo. En esta dirección identifican los siguientes valores que facilitan el aprendizaje: a) tolerancia al error; b) confianza; c) compromiso individual y organizacional; d) compartir la información; apertura para la toma de decisiones; y e) cohesión cultural. Entre los factores que promueven el aprendizaje organizacional mencionan: a) desarrollo de la capacidad de aprendizaje (características del ambiente como contexto) y b) facilitadores (estrategias y procesos que facilitan la generación de entornos de aprendizaje): identidad común; solución de problemas; construcción de equipos; acceso a la información; desarrollo de capacidades individuales; comunicación e inducción.

Por su parte Kale, Singh y Perlmutter (2000) con relación al aprendizaje en el marco de alianzas pusieron el foco en demostrar que cuando las empresas son capaces de lograr capital relacional, basado en la confianza mutua y la interacción a nivel individual entre socios de una alianza, se crea una base para el aprendizaje y de transferencia de conocimientos a través de la interfaz de intercambio. $\mathrm{Al}$ mismo tiempo, esto frena el comportamiento oportunista de los socios de la alianza, lo que impide la fuga de conocimientos. Encuestaron a 212 organizaciones, a partir de un cuestionario conformado por proposiciones a modo de escala de Likert con siete puntos para la respuesta, que luego fueron procesadas con un análisis multivariado, que incluyó los siguientes ítems con relación al aprendizaje y la protección de saberes en alianzas estratégicas.

Las variables independientes son: 1) Capital relacional. Se indaga si existe una interacción cercana entre los actores organizacionales en distintos niveles: si la alianza se caracteriza por respeto; por la mutua confianza; por amistades personales y por la alta reciprocidad entre sus miembros. 2) Manejo del conflicto. Se inquiere sobre la existencia de mecanismos explícitos para resolver los conflictos; si la interacción entre las partes es monitoreada para identificar posibles conflictos; si existe una fuerte comunicación en ambos sentidos para resolver los conflictos; si se pone mucho énfasis en resolver los obstáculos culturales para resolver los conflictos; si las partes se articulan en la resolución de los problemas y conflictos; si las máximas autoridades de los organismos se comprometen en la resolución de conflictos. 3) Existencia de controles (complementariedad y compatibilidad). Se indaga sobre la existencia de una alta complementariedad entre los recursos/capacidades de las partes; si existe una gran similaridad/superposición de las capacidades de cada actor organizacional; si las culturas organizacionales de las organizaciones aliadas son compatibles; si los estilos de gerenciamiento de las organizaciones aliadas son compatibles; si es equitativa o inequitativa la conformación de la estructura de esta alianza; si habían tenido los participantes alianzas anteriores; y si tienen la misma nacionalidad.

Las variables dependientes son 1) aprendizaje, cuyos indicadores son la capacidad de la organización de incorporar nueva o importante información de su socio/s, y de capacidades críticas o habilidades de su/s socio/s; y 2) si la alianza ayudó a la organización a ampliar las capacidades/habilidades existentes con respecto al resguardo y protección del capital social (si la organización pudo proteger sus capacidades centrales o habilidades de su/s socio/s/sus recursos para que no sean apropiados por él).

El estudio concluye que el éxito de las estrategias asociativas depende no solo de factores estructurales, como las condiciones de equidad de las organizaciones involucradas (por ejemplotamaño), sinoespecialmente 
en cómo la organización maneja la alianza luego de su formación, sobre todo en lo concerniente al capital relacional y al manejo de conflictos, puesto que están ligados con el cumplimiento de los objetivos de la alianza como el aprendizaje y la protección de habilidades y capacidades críticas. El aprendizaje, especialmente la adquisición de competencias, que no es sencillo codificar, se logra mejor a través de un continuo e intenso contacto entre los miembros individuales de los socios de la alianza. El capital relacional basado en mutua confianza y respeto fomenta el aprendizaje mediante el fomento de dichos contactos y la voluntad y la capacidad de los socios para participar en un intercambio de información y de «saber» para lograr el aprendizaje recíproco.

En una investigación sobre dos organismos públicos, Encinas Orozco (2014) desarrolla su estudio basándose en el modelo de aprendizaje organizacional MOA (Rodríguez y Trujillo, 2007), formado por dos componentes: el circuito de flujo del conocimiento a través del aprendizaje y los facilitadores que permiten el movimiento del circuito en toda la organización. Este modelo se basa en el circuito de aprendizaje de Kolb y en el de Nonaka y Takeuchi de conversión del conocimiento. Para ello realizó una encuesta a partir de una muestra probabilística a 88 funcionarios en ambas organizaciones. Entre las variables que favorecen el circuito de capacidad de aprendizaje organizacional identifica el liderazgo, el trabajo en equipo, el cambio organizativo, la innovación, la gestión del conocimiento y la estructura organizacional, como los cimientos de una organización que aprende. Concluye que las organizaciones estudiadas presentan una embrionaria disposición al aprendizaje, pero existen barreras tales como la dificultad de compartir conocimientos entre sus miembros, en la medida que subsisten estructuras tradicionales jerarquizadas y poco flexibles que inhiben la innovación y no es suficiente el acceso a tecnología de gestión. La falta de capacitación y los sistemas no adecuados de recompensas y castigos, el escaso uso de herramientas informáticas, la ausencia de foros para compartir buenas experiencias y la falta de monitoreo de las necesidades de los usuarios, son algunos de los obstáculos relacionados con la capacidad de adquirir conocimiento.

Con el objetivo de validar una escala de Likert para caracterizar la capacidad de aprendizaje organizacional Garzón Castillón y Fischer (2010) plantean cuatro variables: a) fuentes del aprendizaje (crisis y problemas, clientes, unidades especializadas, adquisiciones, la competencia, la experiencia, la tecnología, las redes, la historia y los supuestos); b) sujetos del aprendizaje (individuos, equipos, organizaciones e interoganizaciones; c) cultura para el aprendizaje organizacional (concepto de hombre en la organización, el sistema cultural y el clima organizacional); d) condiciones para el aprendizaje organizacional (competencias, estructura, comunidades de práctica, comunidades de compromiso y de aprendizaje y memoria organizacional). En esta misma línea, Castañeda (2015) indaga sobre las condiciones de aprendizaje en tres organizaciones (dos empresas y una organización pública estatal) a partir de las opiniones de sus profesionales sobre sus acuerdos con una escala de Likert y concluye que el aprendizaje organizacional es un proceso que puede facilitarse a partir de la existencia de condiciones organizacionales, tales como la cultura de aprendizaje, la formación, la claridad estratégica y el soporte organizacional.

Como puede observarse, existe una relativa coincidencia entre los distintos autores respecto de las variables utilizadas para el análisis de las condiciones de aprendizaje organizacional, con denominaciones distintas pero muy cercanas, ya que la mayoría remite a los mismos aspectos favorecedores del aprendizaje en las organizaciones. En la tabla que se presenta seguidamente, se identifican cuatro de los autores reseñados y se listan de manera ordenada en horizontal las coincidencias presentes en las variables utilizadas por cada uno organizadas por grandes temas; aspectos vinculados con la cultura organizacional, la respuesta a la solución de problemas, el liderazgo orientado a la construcción e integración de equipos, el desarrollo de las capacidades del personal y de ambientes de aprendizaje, la comunicación y el libre flujo de la información, y apertura al cambio, como oportunidad. 
Tabla 1. Síntesis comparativa de las propuestas de los autores

\begin{tabular}{|c|c|c|c|c|}
\hline \multicolumn{5}{|c|}{ Variables referidas a las condiciones para el AO en distintos autores } \\
\hline $\begin{array}{l}\text { Preocupaciones } \\
\text { centrales }\end{array}$ & Marsick y Waltkins & $\begin{array}{l}\text { Ali, Pascoe y } \\
\text { Warne }\end{array}$ & $\begin{array}{l}\text { Kale, Singh, y } \\
\text { Perlmutter }\end{array}$ & Orozco \\
\hline $\begin{array}{l}\text { Aspectos vinculados } \\
\text { con la cultura } \\
\text { organización }\end{array}$ & $\begin{array}{c}\text { Trabajo diseńado } \\
\text { para el aprendizaje } \\
\text { continuo. } \\
\text { (oportunidades para el } \\
\text { aprendizaje) }\end{array}$ & $\begin{array}{l}\text { Identidad común } \\
\text { Cohesión cultural } \\
\text { Compromiso } \\
\text { individual y } \\
\text { organizacional }\end{array}$ & Capital relacional & \\
\hline $\begin{array}{l}\text { La respuesta a } \\
\text { la solución de } \\
\text { problemas }\end{array}$ & $\begin{array}{l}\text { Empoderamiento de las } \\
\text { personas }\end{array}$ & $\begin{array}{c}\text { Solución de los } \\
\text { problemas }\end{array}$ & $\begin{array}{l}\text { Manejo del } \\
\text { conflicto }\end{array}$ & \\
\hline $\begin{array}{l}\text { Liderazgo y } \\
\text { construcción e } \\
\text { integración de } \\
\quad \text { equipos }\end{array}$ & $\begin{array}{c}\text { Liderazgo para el } \\
\text { aprendizaje y trabajo } \\
\text { colaborativo en equipos }\end{array}$ & $\begin{array}{l}\text { Construcción de } \\
\text { equipos confianza }\end{array}$ & $\begin{array}{l}\text { Existencia de } \\
\text { controles }\end{array}$ & $\begin{array}{l}\text { Liderazgo } \\
\text { Trabajo en equipo }\end{array}$ \\
\hline \multirow{2}{*}{$\begin{array}{c}\text { Desarrollo de } \\
\text { las capacidades } \\
\text { del personal y } \\
\text { de ambientes de } \\
\text { aprendizaje }\end{array}$} & $\begin{array}{l}\text { Promoción de la } \\
\text { indagación y el diálogo } \\
\text { y libertad de expresión }\end{array}$ & $\begin{array}{l}\text { Desarrollo de } \\
\text { las capacidades } \\
\text { personales }\end{array}$ & $\begin{array}{c}\text { Aprendizaje } \\
\text { (capacidad de } \\
\text { incorporar nueva } \\
\text { información) }\end{array}$ & Gestión del conocimiento \\
\hline & $\begin{array}{c}\text { Creación de } \\
\text { oportunidades para el } \\
\text { aprendizaje }\end{array}$ & $\begin{array}{l}\text { Inducción y } \\
\text { enculturación }\end{array}$ & $\begin{array}{c}\text { Crecimiento } \\
\text { de capacidades } \\
\text { existentes }\end{array}$ & \\
\hline \multirow{3}{*}{$\begin{array}{l}\text { La comunicación y } \\
\text { el libre flujo de la } \\
\text { información }\end{array}$} & $\begin{array}{c}\text { Creación de sistemas } \\
\text { para capturar } \\
\text { y compartir el } \\
\text { aprendizaje }\end{array}$ & $\begin{array}{l}\text { Acceso a la } \\
\text { información }\end{array}$ & & \\
\hline & & Comunicación & & \\
\hline & $\begin{array}{l}\text { Conexión de la } \\
\text { organización y su } \\
\text { medio ambiente }\end{array}$ & & & $\begin{array}{c}\text { Estructura de la organización } \\
\text { que ayuda a compartir el } \\
\text { conocimiento }\end{array}$ \\
\hline $\begin{array}{l}\text { Apertura al cambio, } \\
\text { como oportunidad }\end{array}$ & & Tolerancia al error & & Cambio organizativo \\
\hline
\end{tabular}

Fuente: elaboración propia.

\section{EL ÉNFASIS EN LOS PROCESOS, LA GENERACIÓN DE ALIANZAS}

El análisis de la incorporación de conocimiento, a partir de una alianza entre organizaciones del mismo tipo o diferente (por ejemplo, entre la organización pública no estatal y la organización estatal) y la identificación de dimensiones y variables relacionadas con la incorporación de conocimiento a partir de dicha alianza, es un enfoque relevante sobre el aprendizaje.

En esta dirección, Simonin (1999) estudió el proceso de transferencia de conocimiento en alianzas estratégicas en 192 empresas de primer nivel en Estados Unidos, a partir de las respuestas de sus ejecutivos. Concluye que tanto las variables relacionadas con el conocimiento específico (tácito/ complejo) y las variables relacionadas con las características del socio impactan el proceso, y que la ambigüedad del conocimiento es una variable interviniente en los efectos de transferencia del conocimiento. Para ello, organiza su cuestionario en tres dimensiones a) el conocimiento; b) las características del socio de la alianza; y c) los efectos de la alianza (Escala de Likert con valores de 1 a 7).

Respecto de a) conocimiento; se tomaron los siguientes indicadores: 1) Ambigüedad (los procesos tecnológicos del socio son fácilmente transferibles 
a la organización. La asociación entre causas y efectos, salidas y entradas, actividades y productos relacionados con los procesos de conocimientos/ tecnología desarrollados por el socio son claros). 2) Transferencia de conocimientos (la organización ha aprendido sobre tecnologías o procesos mantenidos por sus socios; redujo su dependencia inicial con el socio desde el inicio de la alianza. Los procesos de conocimiento/tecnológicos desarrollados por el socio han sido asimilados por la organización y han contribuido al desarrollo de otros proyectos). 3) Experiencia (independientemente de la alianza la organización tiene un alto nivel de conocimiento de la tecnología/proceso de conocimiento desarrollado por su socio).

Respecto de b) características del socio de la alianza; se tomaron los siguientes indicadores: 1) Especificidad (para el desarrollo de su tecnología el socio tuvo que invertir significativamente en equipo/herramientas especializado/en recursos humanos capacitados). 2) Complejidad (El proceso tecnológico/de conocimiento del socio es producto de muchas interdependientes técnicas, rutinas, individuos y recursos). 3) Tecnologías (La tecnología/ procesos de conocimiento del socio son fácilmente decodificables (planos, instrucciones, formulas. Las tecnologías/procesos de conocimiento que son más explícitos que tácitos). 4) Proteccionismo (el socio tiene procedimientos, rutinas y políticas intencionales para limitar el compartir información relevante concerniente a conocimientos/es muy protector de sus conocimientos/tecnologías). 5) Distancia cultural (la cultura nacional de los socios difiere. Las diferencias de lenguaje son el mayor obstáculo para la comunicación y comprensión con el socio). 6) Distancia organizacional (las prácticas y los mecanismos en las operaciones/la cultura organizacional y el estilo de gerenciamientos son muy similares).

Finalmente, para c) efectos de la alianza, se indagó sobre: 1) Conocimiento colaborativo en orden de ser exitoso en las colaboraciones: el grado en que la organización necesita conocimientos colaborativos en: la identificación de un socio; la selección de un socio; negociación; aspectos legales; comprensión de las implicaciones estratégicas de colaboración; recursos tecnológicos; estimaciones presupuestarias; impuestos; cerrar los acuerdos; personal (reclutar, entrenar, rotar, recompensar); administrar la alianza; construir confianza con el socio; resolver conflictos; renegociar acuerdos iniciales con el socio; transferencias de logística y de recursos; entrenamiento cross cultural; adquisición de conocimientos; protección de conocimientos; repatriación de ganancias o capital; existencia de la alianza. 2) La capacidad de aprendizaje: si se comprometió mucho personal/importantes recursos físicos, financieros, organizacionales y logísticos en el marco de la alianza; y sobre la duración de la alianza (en años).

\section{EL APRENDIZAJE COMO ADAPTACIÓN A LOS CAMBIOS DEL ENTORNO Y LAS ALIANZAS}

Bustinza, Molina y Arias-Aranda (2010) retoman la problemática de las alianzas y el aprendizaje organizacional y destacan los cambios en el entorno como uno de los focos del aprendizaje. Analizan la relación entre los mecanismos organizacionales de aprendizaje, normalmente considerados como capacidades dinámicas, y la flexibilidad operativa de la organización considerada como una capacidad operativa, en el contexto de la medición de sus resultados. Definen el aprendizaje organizacional como un proceso dinámico que permite a la organización adaptarse a los cambios del entorno, favoreciendo la modificación de los patrones y rutinas de comportamiento establecidos. Desde esta perspectiva, las capacidades dinámicas son patrones de conducta con los que la organización de forma sistemática ajusta sus rutinas operativas, a fin de aumentar su eficacia. Por lo tanto, los procesos de aprendizaje pueden ser categorizados como parte de sus capacidades dinámicas, es decir que el proceso de aprendizaje está basado en la repetición, la experimentación y la identificación de nuevas oportunidades. Sostienen que las estrategias seguidas por las empresas en el establecimiento de alianzas de negocios están entre los métodos más eficaces de acceder a nuevos conocimientos, y que cuando se trata de construir una alianza entre empresas, la experiencia previa en similares alianzas juega un papel crucial. De esta manera, el aprendizaje colaborativo es la suma de una serie de habilidades desarrolladas con el tiempo como resultado de colaboraciones con otras organizaciones, y este conjunto de habilidades 
se mejora y aumenta por los efectos de la experiencia. Por lo tanto, puede afirmarse que el conocimiento colaborativo es un tipo específico de conocimiento adquirido por las organizaciones, lo cual tiene un efecto positivo e influye a la hora de entrar en alianzas, tanto en el presente como en el futuro, y tiene un efecto directo sobre el desarrollo de todos los procesos llevados a cabo en la misma organización (Bustinza et al., 2010). Esta capacidad dinámica está, por tanto, directamente relacionada con las rutinas organizativas y los procesos operativos que subyacen a estas rutinas, y el objetivo del estudio fue analizar esta relación y su efecto sobre los resultados empresariales. Para ello, llevaron a cabo un estudio empírico en empresas con más de 20 empleados del sector servicios en España. Los cuestionarios, confeccionados a partir de las escalas (Kale et al., 2000) y (Simonin, 1999) más arriba analizadas, fueron enviados a los altos directivos, cuyas posiciones en la empresa les permitió responder con precisión a las preguntas de una amplia variedad de departamentos (123 cuestionarios válidos).

\section{EL IMPACTO DEL APRENDIZAJE ORGANIZACIONAL EN LA ORGANIZACIÓN}

En su estudio Liao y Wu (2009) ponen el foco sobre la relación entre gestión del conocimiento, aprendizaje organizacional y el rendimiento organizacional y establecen que el aprendizaje organizacional es un mecanismo coordinado y que su gestión afecta la performance organizacional. Identifican diversos componentes del proceso de gestión del conocimiento: captura, transferencia y uso. 1) La captura como el proceso de buscar y adquirir nuevo conocimiento o crear nuevo conocimiento a partir del conocimiento existente a través de la colaboración entre personas y actores involucrados; 2) la transferencia que implica hacer el conocimiento utilizable; y 3) la aplicación como proceso orientado a la utilización del conocimiento. En su estudio trabajaron a partir de una muestra probabilística de 327 empresas de conocimiento intensivo: de alta tecnología industrial (electrónica, aeroespacial y biotecnología) y servicios (educación, comunicación e información) con un cuestionario (Escala de Likert de cinco puntos).

La gestión del conocimiento se orienta a inquirir sobre la existencia de procesos de adquisición de conocimientos sobre clientes/proveedores/ competidores; sobre el uso de la información sobre proyectos para mejorar los subsecuentes proyectos; sobre la existencia de procedimientos para compartir conocimientos con sus asociados; de procesos de evaluación comparativa de rendimiento y de equipos orientados a identificar las mejores prácticas.

La transferencia del conocimiento supone la existencia de procesos para: convertir el conocimiento para el diseño de nuevos productos o servicios; transferir el conocimiento organizacional a los individuos; incorporar el conocimiento de los individuos a la organización; incorporar el conocimiento de los asociados en la organización; incorporar diferentes fuentes y tipos de conocimientos y reemplazar conocimiento obsoleto.

La aplicación del conocimiento indaga sobre la existencia de procesos para: utilizar el conocimiento aprendido a partir de los errores o de experiencias; ponerlo en juego en el desarrollo de nuevos productos/ servicios; usarlo para solucionar nuevos problemas; para mejorar eficiencia; la capacidad para localizar y aplicar conocimiento para cambiar condiciones competitivas; hacer el conocimiento accesible para quienes lo necesitan; establecer conexiones entre las fuentes de conocimiento para solucionar los problemas.

Asimismo, Fernández-Mesa, Alegre-Vidal, y ChivaGómez (2012) retomando el estudio de Bustinza et al. (2010) establecen una relación entre la capacidad de aprendizaje dela organización yla innovación, mediada por algunas variables organizacionales relacionadas con la cultura, tales como la descentralización en la toma de decisiones, la tolerancia al error y las relaciones sociales en la organización. Encuestaron 182 empresas italianas y espańolas para validar esta relación. Operacionalizaron el concepto teórico de capacidad de aprendizaje organizacional en cinco dimensiones, que fueron medidas en 14 ítems a partir de una escala de Likert de siete puntos: capacidad de experimentación, asunción de riesgo, interacción con el entorno, diálogo y toma de decisiones participativa.

López, Ahumada, Olivares y González (2012) indagan sobre el impacto del aprendizaje organizacional en 119 escuelas de la administración municipal chilena y su relación con el desempeńo educativo. Para ello 
administran una escala de Likert de 30 ítems y cinco posibilidades de respuesta. Los resultados obtenidos muestran que existe asociación entre tres condiciones del aprendizaje organizacional (claridad estratégica, cultura de aprendizaje y aprendizaje grupal) y los resultados organizacionales (efectividad, iniciativa, igualdad de oportunidades e integración en las escuelas). La claridad estratégica se relaciona con el reconocimiento de valores y misión organizacionales y cómo el trabajo de sus miembros contribuye a ellos. Definen cultura de aprendizaje como aquella favorable a los procesos de cambio y a la utilización de aprendizajes previos para generar nuevos aprendizajes; es decir procesos que representan como el conocimiento se disemina y distribuye en la organización. Finalmente, el aprendizaje grupal se refiere al aprendizaje colectivo que se da hacia el interior de los grupos de trabajo. A partir de ello concluyen que en la medida que la cultura instalada es favorable a la innovación y al aprendizaje se produce un incremento de los niveles de desempeño educativo.

\section{LA RELACIÓN ENTRE EL APRENDIZAJE INDIVIDUAL, GRUPAL Y ORGANIZACIONAL}

En Brasil (Bido, Godoy, Ferreira, Kenski, y Scartezini, 2011) se estudió el aprendizaje organizacional en el marco de una organización financiera multinacional. Para los autores, el fenómeno del aprendizaje organizacional puede ser estudiado en diferentes niveles que se encuentran conectados, y desde esta perspectiva, analizan la relación que se produce entre los individuos, los grupos y la organización. Se examina la relación entre el aprendizaje individual, grupal y organizacional. Para ello se adaptó el instrumento presentado por Chan (2003) para estudiar el aprendizaje en un hospital australiano, que fue organizado a partir de tres escalas ya existentes y adaptadas para la medición del aprendizaje individual, en los equipos y organizacional. Estuvo compuesto por 41 ítems, siendo nueve dirigidos a la medición del aprendizaje individual, once para el aprendizaje grupal y 21 para el organizacional. Los once ítems que hacen referencia al aprendizaje grupal incluyen comportamientos de aprendizajes internos y externos. El aprendizaje interno se refiere a las formas en que los equipos monitorean la performance frente a los objetivos, obtienen nueva información, testean presupuestos y crean nuevas posibilidades. El aprendizaje externo se refiere a las maneras en que los equipos buscan nueva información y generan el feedback respecto a su trabajo. Los ítems que miden el AO exploran cinco dimensiones: claridad de propósito y misión, compromiso del liderazgo y delegación de poder, prácticas y recompensas, transferencia de conocimiento y equipo de trabajo y solución de problemas en grupo. En su versión final, los 41 ítems fueron mezclados aleatoriamente y las respuestas de los sujetos fueron registradas en una escala Likert con cinco posibilidades de respuesta. El instrumento también cuenta con ocho preguntas demográficas para la caracterización de los encuestados.

Las variables referidas al aprendizaje individual son la frecuencia del aprendizaje y la importancia de los aprendizajes. Las referidas al aprendizaje grupal se centran en el comportamiento interno de aprendizaje (existencia de espacios de discusión para mejorar procesos de trabajo; estrategias para prevenir y aprender de los errores; certificación de procesos grupales de reflexión sobre los procesos de trabajo) y sobre el comportamiento externo de aprendizaje grupal (información que el equipo brinda a la organización sobre lo que planean realizar; si las personas que componen el equipo obtienen información de otras fuentes como los clientes $\mathrm{u}$ otras áreas de la organización). A nivel organizacional se incluyen la claridad del propósito y de la misión organizacionales (oportunidad de autoevaluación respecto del alcance de las metas; la misión de la organización identifica los valores a los cuales todos los miembros deben atenerse); el compromiso del liderazgo y la delegación de poder; las posibilidad de innovar y las recompensas; capacidad de transferencia de conocimiento desde otras organizaciones y, finalmente, la capacidad de trabajar en equipo que involucra a actores de diversas áreas organizacionales para solucionar los problemas en grupo.

\section{DIFICULTADES EN LA GESTIÓN DE LOS APRENDIZAJES ORGANIZACIONALES}

Perlo (2011) estudia el fracaso en la gestión de los cambios y las dificultades en la gestión del 
aprendizaje organizacional, a partir de un abordaje fundamentalmente cualitativo. Enfatiza que para garantizar cambios reales y efectivos debe realizarse una gestión a través del aprendizaje de competencias formativas y organizativas. Desde la revisión de las teorías organizacionales concluye que las organizaciones no son independientes de las personas quelas construyen ylas dirigen, sino que son el resultado de una estructura sociopolítica, como el pensamiento y la acción de los miembros que participan en ellas. Siguiendo con esta perspectiva, la unidad de análisis de este trabajo son las organizaciones, tomando en cuenta el proceso de aprendizaje y cambio, sin apartarse del marco instituido de las mismas. La metodología utilizada privilegia el enfoque fenomenológico-cualitativo a través del estudio de casos. Los indicadores de las variables analizadas del aprendizaje y cambio, a partir de los cuales se diseñaron los instrumentos, fueron: concepto; sujeto; contenido; estrategia, tiempo y espacio. Se trabajó con casos organizacionales y casos grupos de actores sociales. Para el primer caso, se seleccionó una organización educativa y una productora de bienes y servicios. Para el caso de grupos de actores sociales, se trabajó con un grupo de 67 docentes en ejercicio en el sistema educativo; y con 65 profesionales de distintas organizaciones productivas privadas, pertenecientes a distintas posiciones en la estructura organizacional. Los criterios de selección son: el tipo de organización según el aprendizaje formal y no formal; su preocupación por el tema del cambio y la demanda de conocimiento teórico para llevar a cabo este proceso en los colectivos a los que pertenecían. De esta forma, se estudió comparativamente el aprendizaje colectivo, realizando prolongados trabajos de campo. Además de la observación participante, se realizaron entrevistas, cuestionarios, grupos de discusión y series gráficas. Se utilizaron distintas técnicas y estrategias de procesamiento y análisis según la naturaleza de los datos recogidos.

A pesar de ser escasos en el campo, ya existen algunos estudios que buscan contemplar el abordaje metodológico constructivista. Dos de ellos forman parte de una línea de investigación del GAP (grupo de estudios perteneciente al programa de PostGraduación en Administración de la Universidad de Río Grande del Sur). El primero, ya terminado (Salinas, 2001) empleó la grounded theory e investigó los impactos de la difusión de principios y conceptos de $\mathrm{AO}$ en las prácticas de trabajo de los auditores internos de una organización de servicios financieros. El trabajo acompaña, describe, analiza y evalúa, en un proceso de investigación longitudinal, la exploración de métodos de aprendizaje sustentados en el abordaje "aprendizaje experiencial a través de la solución de problemas». La relevancia científica de este trabajo se presenta en tres aspectos: a) todavía son escasos los experimentos que emplean intencional y explícitamente prácticas de aprendizaje organizacional, menos aún en contextos grupales; b) por la utilización de la metodología grounded theory, también poco difundida en Brasil; c) por conseguir compatibilizar el abordaje procesal y técnico (aprendizaje organizacional y organizaciones que aprenden), identificando las barreras que dificultan la obtención del aprendizaje organizacional y las competencias básicas requeridas para la conducción de este proceso.

La revisión crítica de estos antecedentes nos permitieron generar una definición propia del aprendizaje organizacional como un proceso complejo a la vez individual, grupal y organizacional originado en la detección de problemas que afectan el desempeño organizacional, y en el caso más extremo la supervivencia organizacional, a partir del cual las personas y los grupos incorporan y ponen en juego nuevas soluciones/tecnologías/formas de percibir y actuar sobre la realidad en el marco de condiciones específicas de aprendizaje, tales como la cultura organizacional, los estilos de liderazgo, las prácticas comunicacionales, las alianzas con otros sectores/ organizaciones, etc.

\section{VARIABLES SELECCIONADAS PARA LA EXPLORACIÓN DEL APRENDIZAJE ORGANIZACIONAL}

A partir del análisis de los trabajos revisados, se identificaron las siguientes dimensiones con relación al aprendizaje organizacional. 
Tabla 2. Variables orientadas a la exploración del aprendizaje organizacional (AO)

\begin{tabular}{|c|c|c|c|}
\hline Variable & Definición conceptual & Preguntas & Antecedentes \\
\hline Causas del AO & $\begin{array}{l}\text { El motor que dispara el } \\
\text { aprendizaje: una crisis, deseo } \\
\text { de mejorar, la resolución } \\
\text { de un problema, el trabajo } \\
\text { con otros actores dentro de } \\
\text { la organización, el trabajo } \\
\text { con otros actores por fuera } \\
\text { de la organización, otras } \\
\text { organizaciones, la inclusión } \\
\text { de nuevas prácticas de hacer } \\
\text { las cosas el fenómeno de la } \\
\text { distancia cultural en el trabajo } \\
\text { con destinatarios, profesores, } \\
\text { etc. }\end{array}$ & $\begin{array}{l}\text { ¿Cómo se identifican los } \\
\text { problemas? } \\
\text { ¿Cómo se identifican errores } \\
\text { como soluciones propuestas que } \\
\text { no resuelven los problemas? }\end{array}$ & $\begin{array}{l}\text { Ali, Pascoe y Warne (2002) } \\
\text { Bustinza, Molina y Arias- } \\
\text { Aranda (2010) } \\
\text { Liao y Wu (2009) } \\
\text { Perlo (2011) } \\
\text { Salinas (2001) }\end{array}$ \\
\hline $\begin{array}{l}\text { Condiciones del } \\
\mathrm{AO}\end{array}$ & $\begin{array}{l}\text { Son las características de la } \\
\text { organización que hacen posible } \\
\text { la ocurrencia del aprendizaje } \\
\text { organizacional. Por ejemplo, } \\
\text { la existencia de una cultura } \\
\text { abierta a la incorporación } \\
\text { de nuevas prácticas, estilos } \\
\text { de liderazgo democráticos, } \\
\text { apertura a una comunicación } \\
\text { horizontal, la comunicación } \\
\text { eficaz, justicia organizacional, } \\
\text { valoración positiva hacia el } \\
\text { cambio(cultura proactiva ), } \\
\text { etc. }\end{array}$ & $\begin{array}{l}\text { ¿Cómo se logra la identidad } \\
\text { común? } \\
\text { ¿Cómo se logra que los } \\
\text { mecanismos de solución de los } \\
\text { problemas se constituyan en una } \\
\text { oportunidad de aprendizaje? } \\
\text { ¿Cómo se trabaja para que los } \\
\text { miembros de la organización } \\
\text { tengan acceso a la información? } \\
\text { ¿Cómo se trabaja con el } \\
\text { desarrollo de las capacidades } \\
\text { personales? } \\
\text { ¿Cómo se lleva adelante la } \\
\text { comunicación? }\end{array}$ & $\begin{array}{l}\text { Ali, Pascoe y Warne (2002) } \\
\text { Bustinza, Molina y Arias- } \\
\text { Aranda (2010) } \\
\text { Castañeda (2015) } \\
\text { Encinas Orozco (2014) } \\
\text { Garzón Castillón y Fischer } \\
\text { (2010) } \\
\text { Zárate (2007) }\end{array}$ \\
\hline Tipos de AO & $\begin{array}{l}\text { Aquello que se aprende o } \\
\text { se incorpora a los modos de } \\
\text { ser en la organización por } \\
\text { ejemplo: nuevos saberes, } \\
\text { nuevas prácticas, tecnologías, } \\
\text { nuevos valores, se desarrollan } \\
\text { nuevas capacidades, se generan } \\
\text { nuevos productos/servicios. }\end{array}$ & $\begin{array}{l}\text { ¿Qué es lo que aprendieron, } \\
\text { nuevos saberes, nuevas prácticas, } \\
\text { nuevas formas de pensar, nuevas } \\
\text { tecnologías, nuevos...? }\end{array}$ & $\begin{array}{l}\text { Ali, Pascoe y Warne (2002) } \\
\text { Kale, Singh, y Perlmutter. } \\
\text { (2000) } \\
\text { Perlo (2011) } \\
\text { Simonin (1999) }\end{array}$ \\
\hline Proceso de AO & $\begin{array}{l}\text { ¿Cómo se produce el } \\
\text { aprendizaje?, ¿cuál es la historia } \\
\text { de su generación, como es su } \\
\text { origen?, ¿con qué alianzas, qué } \\
\text { impedimentos se presentan } \\
\text { en el camino?, ¿cómo se } \\
\text { superan, cuánto tiempo lleva } \\
\text { su generación? Tiene que } \\
\text { ver con los momentos de la } \\
\text { historia de la organización: } \\
\text { crisis, expansión, extensión de } \\
\text { los procesos, ciclos, etc. }\end{array}$ & $\begin{array}{l}\text { ¿Surgen respuestas innovadoras a } \\
\text { los problemas? } \\
\text { ¿Cómo se trabaja con estas } \\
\text { nuevas soluciones? } \\
\text { ¿Cómo se incorporan en la rutina } \\
\text { diaria? } \\
\text { En la alianza que se realiza con } \\
\text { otras organizaciones ¿Cuáles } \\
\text { son los beneficios para la } \\
\text { organización? } \\
\text { ¿Si surgen conflictos, cómo se } \\
\text { manejan? }\end{array}$ & $\begin{array}{l}\text { Ali, Pascoe y Warne (2002) } \\
\text { Bido, Godoy, Ferreira, } \\
\text { Kenski y Scartezini (2011) } \\
\text { Kale, Singh, y Perlmutter. } \\
\text { (2000) } \\
\text { Perlo (2011) } \\
\text { Simonin (1999) }\end{array}$ \\
\hline
\end{tabular}




\begin{tabular}{|c|c|c|c|}
\hline Variable & Definición conceptual & Preguntas & Antecedentes \\
\hline Fuentes del AO & $\begin{array}{l}\text { A partir de que actividades/ } \\
\text { tareas se incorpora el } \\
\text { aprendizaje: la capacitación, } \\
\text { la experiencia, el trabajo } \\
\text { cotidiano, etc. }\end{array}$ & $\begin{array}{l}\text { ¿Cómo se incorpora el } \\
\text { conocimiento? ¿A través de la } \\
\text { capacitación, el mirar como } \\
\text { hacen otros, los protocolos, etc.? }\end{array}$ & $\begin{array}{l}\text { Ali, Pascoe y Warne (2002) } \\
\text { Liao y Wu (2009) }\end{array}$ \\
\hline El sujeto del $\mathrm{AO}$ & $\begin{array}{l}\text { ¿Quiénes son los que } \\
\text { incorporan el aprendizaje: } \\
\text { los individuos, los grupos, los } \\
\text { equipos, toda la organización? }\end{array}$ & $\begin{array}{l}\text { ¿Quiénes incorporan el } \\
\text { conocimiento? ¿Las personas, } \\
\text { los grupos, los equipos, la } \\
\text { organización? }\end{array}$ & $\begin{array}{l}\text { Ali, Pascoe y Warne (2002) } \\
\text { Bido, Godoy, Ferreira, } \\
\text { Kenski y Scartezini (2011) } \\
\text { Chan (2003) } \\
\text { Kale, Singh, y Perlmutter } \\
\text { (2000) } \\
\text { Perlo (2011) } \\
\text { Simonin (1999) }\end{array}$ \\
\hline $\begin{array}{l}\text { El impacto del } \\
\mathrm{AO}\end{array}$ & $\begin{array}{l}\text { Impacto refiere a los efectos de } \\
\text { los resultados de una acción } \\
{\text { (proyecto, actividad, etc. })^{1} \text {. Los }} \\
\text { efectos refieren a los cambios } \\
\text { en los que se manifiesta el } \\
\text { aprendizaje. }\end{array}$ & $\begin{array}{l}\text { ¿Cuáles son los resultados que } \\
\text { esperaban obtener? } \\
\text { ¿Se logró lo deseado? } \\
\text { ¿Lo pudieron evaluar? } \\
\text { ¿Cuáles fueron los resultados } \\
\text { supuestos/reales/deseados? }\end{array}$ & $\begin{array}{l}\text { Ali, Pascoe y Warne (2002) } \\
\text { Bustinza, Molina, y Arias- } \\
\text { Aranda (2010) } \\
\text { Fernández-Mesa, Alegre- } \\
\text { Vidal, y Chiva-Gómez } \\
\text { (2012) } \\
\text { Kale, Singh, y Perlmutter } \\
\text { (2000) } \\
\text { Liao y Wu (2009) } \\
\text { López, Ahumada, Olivares } \\
\text { y González (2012) } \\
\text { Perlo (2011) } \\
\text { Simonin (1999) }\end{array}$ \\
\hline
\end{tabular}

Fuente: elaboración propia.

\section{A MODO DE CIERRE}

La consideración de que el fenómeno del aprendizaje organizacional es un aspecto valioso de las organizaciones ha impulsado la realización de diversos estudios empíricos, con variedad de enfoques y propuestas. Los estudios revisados, tanto desde abordajes cualitativos como cuantitativos o mixtos, a pesar de sus diferencias, ponen de manifiesto una serie de conceptualizaciones comunes a la hora de arribar al campo y relevar datos. Fue el propósito de este artículo realizar un relevamiento de abordaje cualitativos y cuantitativos del fenómeno, para la elaboración de un cuadro de variables y preguntas de indagación, que contemplara de manera amplia los principales aspectos que componen el fenómeno. Estos aspectos fueron agrupados en las variables de causas del $A O$, condiciones del $A O$, tipos de $A O$, proceso de $A O$, fuentes del $A O$, el sujeto del $A O$, el impacto del $A O$, de manera de reflejar de forma agrupada las preocupaciones presentes en el campo de trabajo actual.

\footnotetext{
${ }^{1}$ Existe un gran debate en torno a la definición de impacto, se toma en este caso la clásica definición de Cohen y Franco (Cohen y Franco, 1997) y no se considera la dimensión de perdurabilidad que comúnmente se asocia al impacto.
} 


\section{REFERENCIAS}

Ali, I.; Pascoe, C. y Warne, L. (2002). Interactions of organizational culture and collaboration in working and learning. Educational Technology and Society, 5(2), 60-68.

Antonacopoulou, E. P. (2006a). The challenges and Prospects of learning-in-practice. Paper presented at the Conference at the University of Warwick, Coventry.

Antonacopoulou, E. P. (2006b). The relationship between individual and organizational learning. New evidence from managerial learning practices. Management learning, 37(4), 455-473. doi:10.1177/1350507606070220

Argyris, C. (1999). Conocimiento para la acción. Una guia para superar los obstáculos del cambio en la organización. Barcelona: Granica.

Bido, D. d. S.; Godoy, A. S.; Ferreira, J. F.; Kenski, J. M. y Scartezini., V. N. (2011). Examinando a relação entre aprendizagem individual, grupal e organizacional em uma instituição financeira. REAd - Revista Eletrônica de Administração, 17, 58-85.

Bustinza, O. F.; Molina, L. M. y Arias-Aranda, D. (2010). Organizational learning and performance: Relationship between the dynamic and the operational capabilities of the firm. African Journal of Business Management, 4(18), 4067-4078.

Castañeda, D. I. (2015). Condiciones para el aprendizaje organizacional. Estudios Gerenciales, 31(134), 62-67. http://doi.org/10.1016/j. estger.2014.09.003

Chan, C. C. A. (2003). Examining the relationships between individual, team and organizational learning in an Australian hospital. Learning in Health and Social Care, 2(4), 223-235. doi:10.1046/j.1473-6861.2003.00057.x

Encinas Orozco, F. (2014). Orientación de las organizaciones públicas al aprendizaje organizacional . El caso de los organismos descentralizados en el Estado de Sonora, México, Estudios Gerenciales 30, 10-17.

Fernández-Mesa, A.; Alegre-Vidal, J. y Chiva-Gómez, R. (2012). Orientación emprendedora, capacidad de aprendizaje organizativo y desempeño innovador. Journal of Technology Management and Innovation, 7(2), 157-170.

Garzón Castrillón, M. A. y Fischer, A. L. (2010). El aprendizaje organizacional, prueba piloto de instrumentos tipo Likert. Forum Empresarial, 15(1), 65-101.

Gore, E. y Mazzini, M. V. (2010). Hacer visible lo invisible. Buenos Aires: Granica.

Kale, P.; Singh, H. y Perlmutter, H. (2000). Learning and protection of proprietary assets in strategic alliances: Building relational capital. Strategic Management Journal, 21, 217-237.

Liao, S.-h. y Wu, C. C. (2009). The relationship among knowledge management, organizational learning, and organizational performance. International Journal of Business and Management, 4(4), 6474. doi:10.1108/09696470410521628

López, V.; Ahumada, L.; Olivares, R. y González, Á. (2012). Escala de medición del aprendizaje organizacional en centros escolares. Psicothema, 24(2), 323-329.

Marsick, V.J. y Watkins, K.E. (2003). Demostrating the value of an organizations's learning culture: Dimensions of the Learning Organization Questionnaire. Advances in Developing Human Resources, 5(2), 132-151.

Moore, M. (2006). Creando valor público a través de asociaciones público privadas. Revista del CLAD, Reforma y Democracia.

Pereira, L. C. B. y Grau, N. C. (1998). Lo público no estatal en la reforma del Estado. Buenos Aires: Paidós-Clad.

Perlo, C. (2011). Procesos de cambio colectivo, nuevos marcos de comprensión para aprender el holomovimiento. Revista Venezolana de Informacion, Tecnología y Conocimiento, 81-96.

Ramírez, J. A. C. y Zapata, J. P. (2011). El aprendizaje organizacional: reflexión desde la investigación aplicada en el grupo de estudios empresariales. Cuadernos de Administración, 24(39), 29-36.

Rocha, M. I. M. (2013). Gestión del conocimiento y su importancia en las organizaciones. TRILOGÍA. Ciencia, Tecnología y Sociedad 9, 25-35. 
Rodríguez, A. M. E. y Cedeño, B. E. (2012). La innovación entre dos manos: la invisible del mercado y la visible del Estado. TRILOGÍA. Ciencia, Tecnología y Sociedad 6, 13-30.

Rodríguez J. M. y Trujillo J. C. (2007) ¿Las universidades son organizaciones que aprenden adecuadamente? Universia Business Review, 3(15),100-119 Disponible en: http://ubr. universia.net/pdfs/ UBR0032007100.pdf

Rostro Hernández, P. E. y Solís Hernández, O. (2015). Conceptualizando el diálogo entre cultura y aprendizaje organizacional. TRILOGÍA. Ciencia, Tecnología y Sociedad, 7(12), 95-103.

Rúas, R. y Antonello, C. S. (2003). Repensando os referenciais analíticos em aprendizagem organizacional: uma alternativa para análise multidimensional. Revista de Administração Contemporânea, 7(3), 203-212. http://doi. org/10.1590/S1415-65552003000300011
Salinas, J. L. (2001). Impactos da aprendizagem organizacional nas práticas de auditoria interna: um estudo no banco do Brasil (Programa de PósGraduação em Administração), Universidadade Federal do Rio Grande do Sul, Porto Alegre.

Schein, E. (1992). Cultura organizacional y liderazgo. San Francisco: Jossey y Bass.

Senge, P. (2005). La Quinta Disciplina. Cómo impulsar el aprendizaje en la organización inteligente. Barcelona: Granica.

Simonin, B. L. (1999). Ambiguity and the process of knowledge transfer in strategic alliances. Strategic management journal, 20(7), 595-623.

Watkins K. E. y Marsick, V.J. (1998). Dimensions of the Learning Organization Questionnaire. Warwick, RI: Partners for the Learning Organizatio.

Zárate, A. S. (2007). La «organización que aprende» y su aporte al proceso de cambio. Persona, (10), 29-47. 